\title{
Response to 'Peptidyl arginine deiminase type IV (PADI4) haplotypes interact with shared epitope regardless of anti-cyclic citrullinated peptide antibody or erosive joint status in rheumatoid arthritis: a case control study'
}

\author{
Katsunori Ikari", Koichiro Yano, Shinji Yoshida, Atsuo Taniguchi, Hisashi Yamanaka and Shigeki Momohara
}

Rheumatoid arthritis (RA) is a complex polygenic disease characterized by progressive joint destruction. Anti-citrullinated peptide antibody (ACPA) is the most specific autoantibody for RA. Genetic polymorphisms in the PADI4 gene, encoding citrullinating enzyme peptidylarginine deiminase 4 (PADI4), have been associated with susceptibility to RA [1,2]. They have also been reported to be associated with radiographic joint destruction in patients with RA [3,4]. We focused on ACPA-negative RA patients to investigate whether a PADI4 polymorphism is associated with joint damage in ACPA-negative patients.

DNA samples from 122 Japanese ACPA-negative RA patients were used for the study; $81.1 \%$ were female, $51.6 \%$ were rheumatoid factor (RF)-positive, and the mean age was 55 years. Sharp/van der Heijde score of the hands at a 5-year disease duration, which represents joint damage, was scored and log-transformed as described elsewhere [4]. Single-nucleotide polymorphism (SNP) rs2240340 was selected and genotyped by using a TaqMan method as described elsewhere [4]. The genetic risk of joint damage associated with rs2240340 was assessed by multiple regression analysis adjusted for HLA-DRB1 shared-epitope alleles and RF that are thought to be associated with joint damage in patients with RA [4].
The PADI4 SNP was significantly associated with radiographic joint destruction in the ACPA-negative RA patients in a recessive model $(P=0.0287)$ (Table 1 and Figure 1). The overall genotyping success rate was $99.2 \%$ and the genotype concordance rate was $100 \%$ as assessed by duplicate samples. Although the sample size is one of the major limitations to the study of ACPA-negative patients because of the high positivity of ACPA (up to 90\%) in RA populations, we were able to collect DNA samples from 122 ACPA-negative RA patients with radiographic data.

PADI4 encodes citrullinating enzymes that may play an important role in ACPA formation. It has been shown that PADI4 alleles were associated with the presence of ACPA in patients with RA [1]. In the present study, we have shown that PADI4 polymorphism contributes to joint destruction in ACPA-negative RA patients. A recent study has suggested that PADI4 gene contributes to the development of RA, regardless of ACPA status [3]. We have also reported that the PADI4 risk allele has an impact on joint damage after adjustment for ACPA status [4]. The PADI4 gene is likely to play a role in the disease progression of RA in addition to its role in ACPA formation. The results of this study provide important knowledge of the risks on progressive joint damage in patients with RA.

* Correspondence: kikari@twmu.ac.jp

Institute of Rheumatology, Tokyo Women's Medical University, 10-22 Kawada, Shinjuku, Tokyo 162-0054, Japan 
Table 1 Association of PADI4 SNP (recessive model) with Sharp/van der Heijde score of hands at 5-year disease duration in ACPA-negative RA patients

\begin{tabular}{lll}
\hline Factor & Beta $(\mathbf{9 5} \% \mathrm{Cl})$ & $\boldsymbol{P}$ value \\
\hline rs2240340, PAD/4 & $0.72(0.08-1.36)$ & 0.0287 \\
HLA-DRB1 SE & $0.36(0.01-0.73)$ & 0.0587 \\
RF & $0.54(0.05-1.02)$ & 0.0316
\end{tabular}

ACPA, Anti-citrullinated peptide antibody; $\mathrm{Cl}$, Confidence interval; PADI4, Peptidyl arginine deiminase type IV; RA, Rheumatoid arthritis; RF, Rheumatoid factor; SE, Shared epitope; SNP, Single-nucleotide polymorphism.

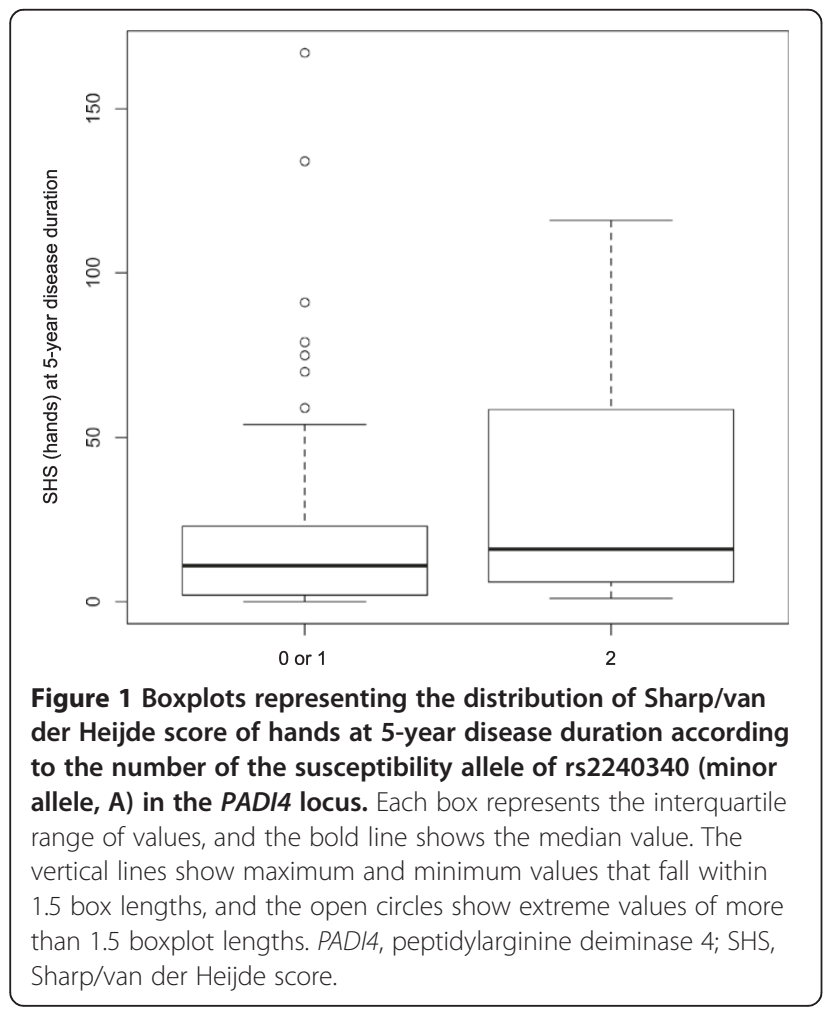

\section{Abbreviations}

ACPA: Anti-citrullinated peptide antibody; PADI4: Peptidylarginine deiminase 4; RA: Rheumatoid arthritis; RF: Rheumatoid factor; SNP: Single-nucleotide polymorphism.

\section{Competing interests}

The authors declare that they have no competing interests.

\section{Acknowledgments}

This work was supported by grants provided by the Japanese Ministry of Education, Culture, Sports, Science and Technology Grant-in-Aid for Scientific Research (to KI) and the Japanese Ministry of Health, Labour and Welfare (to SM and HY). We thank all DNA donors for making this study possible. We appreciate the members of Institute of Rheumatology of the Tokyo Women's Medical University for their effort in the Institute of Rheumatology Rheumatoid Arthritis (IORRA) cohort. We are grateful to Noriko likuni for her assistance in preparing the manuscript and to Yuta Kochi and Akari Suzuki for their advice.

Published online: 27 August 2014

\section{References}

1. Suzuki A, Yamada R, Chang X, Tokuhiro S, Sawada T, Suzuki M, Nagasaki M, Nakayama-Hamada M, Kawaida R, Ono M, Ohtsuki M, Furukawa H, Yoshino S, Yukioka M, Tohma S, Matsubara T, Wakitani S, Teshima R, Nishioka Y, Sekine A, lida A, Takahashi A, Tsunoda T, Nakamura Y, Yamamoto K: Functional haplotypes of PADI4, encoding citrullinating enzyme peptidylarginine deiminase 4 , are associated with rheumatoid arthritis. Nat Genet 2003, 34:395-402.

2. Okada Y, Wu D, Trynka G, Raj T, Terao C, Ikari K, Kochi Y, Ohmura K, Suzuki A, Yoshida S, Graham RR, Manoharan A, Ortmann W, Bhangale T, Denny JC, Carroll RJ, Eyler AE, Greenberg JD, Kremer JM, Pappas DA, Jiang L, Yin J, Ye L, Su DF, Yang J, Xie G, Keystone E, Westra HJ, Esko T, Metspalu A, et al: Genetics of rheumatoid arthritis contributes to biology and drug discovery. Nature 2014, 506:376-381.

3. Bang SY, Han TU, Choi CB, Sung YK, Bae SC, Kang C: Peptidyl arginine deiminase type IV (PADI4) haplotypes interact with shared epitope regardless of anti-cyclic citrullinated peptide antibody or erosive joint status in rheumatoid arthritis: a case control study. Arthritis Res Ther 2010, 12:R115.

4. Suzuki T, Ikari K, Yano K, Inoue E, Toyama Y, Taniguchi A, Yamanaka H, Momohara S: PADI4 and HLA-DRB1 are genetic risks for radiographic progression in RA patients, independent of ACPA status: results from the IORRA cohort study. PloS One 2013, 8:e61045.

doi:10.1186/s13075-014-0422-3

Cite this article as: Ikari et al:: Response to 'Peptidyl arginine deiminase type IV (PADI4) haplotypes interact with shared epitope regardless of anti-cyclic citrullinated peptide antibody or erosive joint status in rheumatoid arthritis: a case control study'. Arthritis Research \& Therapy 2014 16:422 